\title{
MT1-MMP promotes the proliferation and invasion of gastric carcinoma cells via regulating vimentin and E-cadherin
}

\author{
BO LI ${ }^{1,2}$, GUOCHUN LOU ${ }^{3}$ and JUYING ZHOU ${ }^{1}$ \\ ${ }^{1}$ Department of Radiotherapy, The First Affiliated Hospital of Suzhou University, Suzhou, Jiangsu 215006; \\ Departments of ${ }^{2}$ Radiology and ${ }^{3}$ Gastroenterology, Zhejiang Hospital, Hangzhou, Zhejiang 310000, P.R. China
}

Received January 20, 2018; Accepted October 23, 2018

DOI: $10.3892 / \mathrm{mmr} .2019 .9918$

\begin{abstract}
The present study aimed to explore the possible effects of membrane-type 1 matrix metalloproteinase (MT1-MMP) on gastric carcinoma cells proliferation and invasion. Immunohistochemistry analysis was conducted to measure MT1-MMP expression level in 15 patients with gastric carcinoma. Subsequently, recombinant short hairpin RNA (shRNA) vectors targeting MT1-MMP were constructed to silence the expression of MT1-MMP in gastric carcinoma cells. Then, the inhibitive efficiency was verified via reverse transcription quantitative polymerase chain reaction (RT-qPCR) and western blot analysis. The effects of MT1-MMP silencing on cell proliferation and invasion were detected through Cell Counting Kit- 8 test and Transwell assays. The expression levels of vimentin and epithelial cadherin (E-cadherin) were detected by RT-qPCR. The immunohistochemistry analysis results revealed that MT1-MMP expression in gastric carcinoma tissues was markedly overexpressed compared with non-cancerous adjacent tissues. The MT1-MMP expression level in cancer-derived cell line AGS cells was also significantly increased compared with that in non-cancer-derived GES-1 cells. In addition, the MT1-MMP expression level in AGS cells was significantly decreased via shRNA transfection. Cell proliferation and invasion were markedly inhibited following knockdown of MT1-MMP level in AGS cells. These inhibitory effects were associated with the decreased expression of vimentin and increased expression of E-cadherin. MT1-MMP was overexpressed in gastric carcinoma cells, and silencing of MT1-MMP inhibited the proliferation and invasion of cells via regulating the expression of vimentin and E-cadherin.
\end{abstract}

Correspondence to: Dr Juying Zhou, Department of Radiotherapy, The First Affiliated Hospital of Suzhou University, 188 Shizi Street, Gusu, Suzhou, Jiangsu 215006, P.R. China

E-mail: junnyZ_3470@163.com

Key words: gastric carcinoma, membrane-type 1 matrix metalloproteinase, invasion, vimentin, E-cadherin

\section{Introduction}

Gastric carcinoma, a major cause of cancer-associated mortality worldwide, remains a concern for clinicians and the scientific community (1). The majority of patients with gastric carcinoma are diagnosed at advanced stages and have poor outcomes, with susceptibility to invasion and metastasis $(2,3)$. Unfortunately, the development of techniques for the treatment and diagnosis of gastric carcinoma in previous years have only made a modest contribution towards improving the prognosis of gastric carcinoma, in particular for patients with advanced-stage disease (4-6). Therefore, the exploration of novel approaches for the early diagnosis and management of gastric carcinoma is urgently required.

The matrix metalloproteinases (MMPs) family was demonstrated to be overexpressed in multiple types of cancer and to serve essential roles in the development and invasion of cancer (7-9). Accumulating evidence has indicated that several MMPs regulate the migration and invasion of cancer cells by promoting epithelial-mesenchymal transition (EMT) of cancer cells, including MMP-3, MMP-8 and MMP-14 (10-12). Membrane-type 1 matrix metalloproteinase (MT1-MMP) was demonstrated to be expressed at abnormally high levels in cancerous tissues from patients with gastric cancer (13). A previous study demonstrated that the overexpression of MT1-MMP was associated with poor prognoses of patients with gastric carcinoma, including increased tumor invasion, metastasis, advanced Tumor-Node-Metastasis stages and worse overall survival (14). Inhibition of MT1-MMP expression may inhibit cell migration, invasion, proliferation and angiogenesis $(13,15)$. However, the specific mechanisms of MT1-MMP in gastric carcinoma remain unclear.

In the present study, the expression of MT1-MMP was determined in gastric carcinoma clinical samples. In addition, MT1-MMP expression was suppressed using a short hairpin RNA (shRNA) technique followed by proliferation and invasion assays. Using these analyses, the present study aimed to provide useful new information concerning the underlying mechanisms of gastric carcinoma.

\section{Materials and methods}

Tissue samples. Clinic tissue samples were obtained from 15 patients who underwent gastric carcinoma resection at 
the First Affiliated Hospital of Suzhou University (Suzhou, China). Concomitantly, normal gastric tissue samples were collected to serve as controls, which were sourced from a site distant the cancerous lesion $(\geq 5 \mathrm{~cm}$ ) and blindly confirmed by two experienced pathologists. Among the 15 patients, 5 were females and 10 were males. The age of patients ranged from 49-81, and the average age was 61.267 years old. According to the Goseki classification (16), a total of 5 tumors were classed as moderately differentiated adenocarcinomas, 2 were moderately-poorly differentiated, and 8 were poorly differentiated. The present study was approved by the Research Ethics Committee of the First Affiliated Hospital of Suzhou University and was performed according to ethical standards of the Declaration of Helsinki. All participants provided written consent for their clinical information to be used for scientific research.

Immunohistochemistry and hematoxylin-eosin staining $(H \& E)$. Immunohistochemical staining and $\mathrm{H} \& \mathrm{E}$ staining were performed on $4 \%$ paraformaldehyde-fixed (for $24 \mathrm{~h}$ at room temperature), paraffin-embedded tissue sections. For immunohistochemistry, the sections were blocked with $10 \%$ goat serum (OriGene Technologies, Inc., Beijing, China) at $37^{\circ} \mathrm{C}$ for $1 \mathrm{~h}$. MT1-MMP expression was detected with anti-MT1-MMP antibody (cat. no. ab51074; Abcam, Cambridge, UK; dilution, 1:100), followed by horseradish peroxidase-conjugated goat anti-rabbit $\operatorname{IgG}(\mathrm{H}+\mathrm{L})$ (cat. no. 111-035-045; Jackson ImmunoResearch Laboratories, Inc., West Grove, PA, USA) antibody at a dilution of 1:400. Assessment of immunohistochemical staining was performed as described previously by Pang et al (17) and Di Martino et al (18). For H\&E staining, sections were stained with hematoxylin solution $(0.2 \%)$ for $4 \mathrm{~min}$, followed by eosin solution $(0.5 \%)$ for $90 \mathrm{sec}$ at room temperature.

Cell culture. The human gastric cancer AGS cell line and normal gastric epithelial GES-1 cell line were purchased from The Cell Bank of Type Culture Collection of Chinese Academy of Science (Shanghai, China). GES-1 cells were cultured in RPMI-1640 medium (Gibco; Thermo Fisher Scientific, Inc., Waltham, MA, USA) supplemented with $10 \%$ fetal bovine serum (FBS; Gibco; Thermo Fisher Scientific, Inc.), 1\% streptomycin and $1 \%$ penicillin (Gibco; Thermo Fisher Scientific, Inc.). AGS cells were cultured in Dulbecco's modified Eagle's medium (DMEM; Gibco; Thermo Fisher Scientific, Inc.) supplemented with $10 \%$ FBS, $1 \%$ streptomycin and $1 \%$ penicillin. All cells were maintained in a $\mathrm{CO}_{2}$ incubator (Thermo Fisher Scientific, Inc.) with $5 \% \mathrm{CO}_{2}$ at $37^{\circ} \mathrm{C}$.

Construction of shRNA vector and cell transfection. A total of 4 shRNA sequences against MT1-MMP were designed, synthesized and inserted (50 ng) into pLKO.1-puro vector (Sigma-Aldrich; Merck KGaA, Darmstadt, Germany) through Age I (ACCGGT) and Eco RI (GAATTC) restriction enzyme cutting sites. The sequences of the 4 oligonucleotides are summarized in Table I. A scrambled shRNA negative control (NC) sequence (shRNA-NC; Sangon Biotech Co., Ltd., Shanghai, China) was generated through complementary pairs of primers: shNC- forward, 5'-CCGGGTTCTCCGAACGTG TCACGTCAAGAGATTACGTGACACGTTCGGAGAATT
TTTTGGTACC-3' and shNC-reverse, 3'-CAAGAGGCTTGC ACAGTGCAGTTCTCTAATGCACTGTGCAAGCCTCTT AAAAAACCATGGTTAA-5' and used as the negative control. Different shMT1-MMP $(3 \mu \mathrm{g})$ and negative control shRNA vectors $(3 \mu \mathrm{g})$ were transduced into AGS cells by lentivirus. Briefly, the recombinant plasmids were transfected into 293T cells by lentiviruses using a Lipofectamine 2000 transfection kit (Invitrogen; Thermo Fisher Scientific, Inc.). Then, 293T cells were cultured in DMEM (Sigma-Aldrich; Merck KGaA) with $10 \%$ FBS for $24 \mathrm{~h}$. Following replication, the viruses were harvested for the infection of the AGS cells. Subsequent experiments were then performed after $48 \mathrm{~h}$ transfection.

Reverse transcription quantitative polymerase chain reaction (RT-qPCR) assay. Total RNA was isolated from GES-1 and AGS cells using the total RNA extraction reagent RNAiso Plus (Takara Biotechnology Co., Ltd., Dalian, China) according to the manufacturer's protocol. cDNA was generated from RNA using PrimeScript RT Master Mix (Takara Biotechnology Co., Ltd.). RT-qPCR was performed with SYBR Premix EX Taq (Takara Biotechnology Co., Ltd.) as previously described (13). Briefly, reactions were performed with the following components: $5 \mu \mathrm{l}$ 2X SYBR Premix EX Taq, $3.4 \mu \mathrm{l}$ cDNA and $10 \mu \mathrm{M}$ primers, in a final volume of $10 \mu \mathrm{l}$. $\beta$-actin was used as the control. The PCR thermocycler conditions were as follows: $50^{\circ} \mathrm{C}$ for $3 \mathrm{~min}$ and $95^{\circ} \mathrm{C}$ for $3 \mathrm{~min}$, followed by 30 cycles of $95^{\circ} \mathrm{C}$ for $10 \mathrm{sec}$ and $60^{\circ} \mathrm{C}$ for $30 \mathrm{sec}$, and finally $72^{\circ} \mathrm{C}$ for $5 \mathrm{~min}$. The relative quantities of mRNAs were estimated using the $2^{-\Delta \Delta \mathrm{Cq}}$ method (19). The gene primers used are summarized in Table II.

Western blot analysis. GES-1 and AGS cells were lysed with radioimmunoprecipitation assay lysis buffer III (Sangon Biotech Co., Ltd., Shanghai, China), and concentration was quantified using a bicinchoninic acid protein assay kit (Pierce; Thermo Fisher Scientific, Inc.). A total of $20 \mu \mathrm{g}$ protein was loaded in each lane of a $10 \%$ SDS-PAGE gel and separated by electrophoresis. Then, the proteins were transferred onto a polyvinylidene fluoride (PVDF) membrane followed by blocking with $5 \%$ skim milk for $1 \mathrm{~h}$ at room temperature. Following washing with PBS $+0.1 \%$ Tween-20 (PBST) buffer for $5 \mathrm{~min}$, PVDF membranes were incubated with primary antibodies against MT1-MMP (dilution, 1:200; cat. no. ab51074; Abcam) and $\beta$-actin (dilution, 1:1,000; cat. no. 4967; Cell Signaling Technology, Inc., Danvers, MA, USA) at $4^{\circ} \mathrm{C}$ overnight. Then, membranes were incubated for $2 \mathrm{~h}$ at room temperature with horseradish peroxidase-conjugated goat anti-rabbit $\operatorname{IgG}(\mathrm{H}+\mathrm{L})$ (cat. no. ab0101; ProteinTech Group, Inc., Chicago, IL, USA; dilution, 1:1,000) secondary antibodies. Subsequent to washing 3 times with PBST, proteins were detected by chemiluminescence (ECL; EMD Millipore, Billerica, MA, USA), and the expression was quantified by Image Pro Plus 6.0 software (Media Cybernetics, Inc., Rockville, MD, USA).

Proliferation analysis. A Cell Counting Kit-8 (CCK-8; Beyotime Institute of Biotechnology, Shanghai, China) was used to evaluate the proliferation ability and viability of AGS cells. The transfected AGS cells were resuspended, and $100 \mu 1$ AGS cells were seeded in 96 -well plates $\left(2 \times 10^{3}\right.$ cells/well). 
Table I. Sequences of four short hairpin RNAs (shRNA)

\begin{tabular}{ll}
\hline Oligonucleotides & Primer sequences \\
\hline shRNA-1 & 5'-ACCGGTGGGTCTCAAATGGCAACATAATTCAAGAG \\
shRNA-2 & ATTATGTTGCCATTTGAGACCCTTTTTTGAATTC-3' \\
& 5'-ACCGGTGGGAGATGTTTGTCTTCAAGGTTCAAGAGAC \\
shRNA-3 & CTTGAAGACAAACATCTCCCTTTTTTGAATTC-3' \\
shRNA-4 & 5'-ACCGGTGCGGGTGAGGAATAACCAAGTTTC \\
& AAGAGAACTTGGTTATTCCTCACCCGCTTTTTTGAATTC-3' \\
& 5'-ACCGGTGGAAACAAGTACTACCGTTTCTTCAAGAG \\
\hline
\end{tabular}

shRNA, short hairpin RNA. Italics represent the restriction enzyme cutting sites.

Table II. Sequences of primers used in the reverse transcription quantitative polymerase chain reaction assay.

\begin{tabular}{ll}
\hline Genes & \multicolumn{1}{c}{ Primer sequences } \\
\hline MT1-MMP & F: 5'-GGCTACAGCAATATGGCTACC-3' \\
& R:5'-GATGGCCGCTGAGAGTGAC-3' \\
Vimentin & F: 5'-GGACCAGCTAACCAACGACA-3' \\
& R:5'-AAGGTCAAGACGTGCCAGAG-3' \\
E-cadherin & F: 5'-CTTTGACGCCGAGAGCTACA-3' \\
& R:5'-TCGACCGGTGCAATCTTCAA-3' \\
3-actin & F: 5'-TGACAACTTTGGTATCGTGGAAGG-3' \\
& R:5'-AGGCAGGGATGATGTTCTGGAGAG-3' \\
\hline F, forward; R, reverse; MIT1-MMP, membrane type 1 matrix metal- \\
loproteinase; E-cadherin, epithelial cadherin.
\end{tabular}

AGS cells were cultivated for $24,48,72$ and $96 \mathrm{~h}$, then $10 \mu \mathrm{l}$ CCK- 8 solution was added at each time point and cultivated for $2 \mathrm{~h}$ at $37^{\circ} \mathrm{C}$. The optical density values were evaluated at $450 \mathrm{~nm}$ by microplate reader (Epoch; BioTek Instruments, Inc., Winooski, VT, USA). All experiments were performed in quintuplicate.

Transwell analysis. Matrige ${ }^{\circledR}$ (Corning Incorporated, Corning, NY, USA) was diluted 1:6 by serum-free culture media, added to the upper Transwell chamber and incubated for $1 \mathrm{~h}$ at $37^{\circ} \mathrm{C}$ prior to use. The transfected AGS cells were cultivated for $12 \mathrm{~h}$ and then the culture media was refreshed. After $48 \mathrm{~h}$ incubation, the cells were resuspended in serum-free media, counted and seeded into the upper chamber with $100 \mu \mathrm{l}$ cell suspension for incubation. The bottom chamber was loaded with $500 \mu 1$ DMEM with $20 \% \mathrm{FBS}$. After $24 \mathrm{~h}$ incubation at $37^{\circ} \mathrm{C}$, media for AGS cells in the upper chamber was removed and washed twice by PBS. The cells were fixed in $4 \%$ formaldehyde at room temperature for $15 \mathrm{~min}$, washed and stained by $0.01 \%$ crystal violet at room temperature for $20 \mathrm{~min}$. Cells on the upper surface were removed by cotton swabs and the invasive cells
Table III. Hematoxylin and eosin analysis of tissue sections from 15 patients

\begin{tabular}{lc}
\hline Clinicopathological features & Number \\
\hline Sample type & 15 \\
Normal & 15 \\
Gastric carcinoma & \\
Sex & 10 \\
Male & 5 \\
Female & \\
Age, years & $49-81$ \\
Range & 61.267 \\
Mean & \\
Tumor invasion depth & 6 \\
Serosal layer & 1 \\
Muscular layer & 1 \\
Superficial muscular layer & 2 \\
Deep muscular layer & 2 \\
Tunica muscularis mucosae & 3 \\
Tela submucosa & \\
Tumor differentiation & \\
Moderate & 5 \\
Moderate-poor & 2 \\
Poor & \\
\hline
\end{tabular}

were counted under an inverted microscope (IX73; Olympus Corporation, Tokyo, Japan) at x200 magnification.

Statistical analysis. SPSS statistical software 19.0 (IBM Corp., Armonk, NY, USA) was used to analyze the data. Measurement data were presented as mean \pm standard deviation. An unpaired Student's t-test was used to analyze the statistical significance between two groups. One- and two-way analysis of variance were used to compare data between three or more groups, followed by Bonferroni's post-hoc test. Enumeration data were presented as percentage, and analyzed by Chi-square test. 
Table IV. Expression of MT1-MMP proteins in gastric carcinoma tissues and non-cancerous adjacent tissues.

\begin{tabular}{|c|c|c|c|c|c|c|}
\hline \multirow[b]{2}{*}{ Groups } & \multirow{2}{*}{$\begin{array}{l}\text { Total } \\
\text { cases }\end{array}$} & \multicolumn{5}{|c|}{ MT1-MMP expression (\%) } \\
\hline & & - & + & ++ & +++ & P-value \\
\hline Gastric carcinoma & 15 & $0(0.00)$ & $3(20.00)$ & $8(53.33)$ & $4(26.67)$ & $<0.01$ \\
\hline Control & 15 & $0(0.00)$ & $10(66.67)$ & $5(33.33)$ & $0(0.00)$ & - \\
\hline
\end{tabular}

MIT1-MMP, membrane type 1 matrix metalloproteinase; -, negative; +, weak positive; ++, moderately positive; +++, intensely positive.

$\mathrm{P}<0.05$ was considered to indicate a statistically significant difference. All experiments were conducted in triplicate.

\section{Results}

$H \& E$ analysis. The H\&E analysis results of tissue sections from 15 patients are summarized in Table III. In accordance with the clinical diagnosis of gastric carcinoma, all patients exhibited different levels of tumor differentiation and invasion. In the present study, the results of the H\&E staining from a representative patient is presented in Fig. 1.

Immunohistochemical analysis of MT1-MMP expression. Immunohistochemical analysis was performed to detect MT1-MMP expression. The immunohistochemical analysis results revealed that MT1-MMP expression was observed in all patients with gastric carcinoma (Table IV). Notably, the expression of MT1-MMP in gastric carcinoma tissues was prominently overexpressed compared with non-cancerous adjacent tissues (Fig. 2 and Table IV; $\mathrm{P}<0.01$ ). Concomitantly, MT1-MMP was predominantly localized in the cytoplasm of the tumor cells in gastric carcinoma (Fig. 2A).

Verification and selection of shRNA. A total of 4 interfering vectors targeting MT1-MMP were constructed and transfected into AGS cells. To select the most effective shRNA sequence, the mRNA expression level of MT1-MMP in transfected AGS cells was measured by RT-qPCR. ShRNA-2 exhibited the most significant level of interference $(0.601 \pm 0.026$; Fig. 3A; $\mathrm{P}<0.001)$. The western blot analysis results also indicated that the effect of shRNA-2 $(0.750 \pm 0.004)$ on the expression level of MP1-MMP was significant (Fig. 3B and C; $\mathrm{P}<0.001$ ). According to these data, shRNA-2 was used to transfect AGS cells and to evaluate the effect of MT1-MMP.

Expression of MT1-MMP in GES-1 and AGS cells. The expression level of MT1-MMP in GES-1 cells and AGS cells was evaluated. Compared with the GES-1 cells, the gastric adenocarcinoma-derived AGS cells exhibited significantly increased expression levels of MT1-MMP at the mRNA (Fig. 4A; $\mathrm{P}<0.001$ ) and protein levels (Fig. 4B and $\mathrm{C} ; \mathrm{P}<0.001$ ).

Inhibition of AGS cells proliferation. A CCK-8 assay was performed to explore the role of MT1-MMP in the proliferation of AGS cells. The results of the CCK-8 assay revealed that inhibiting MT1-MMP by shRNA-2 significantly decreased the proliferation rate of AGS cells compared to the blank-transfected cells at $72 \mathrm{~h}(\mathrm{P}<0.001)$ and $96 \mathrm{~h}$ $(\mathrm{P}<0.01 ;$ Fig. 5). These results suggested that MT1-MMP may promote the proliferation of gastric carcinoma cell.

Inhibition of MT1-MMP resulted in reduced invasion ability. To assess the effect of MT1-MMP on gastric carcinoma cells, the invasion abilities of AGS cells were assessed using a Transwell invasion assay following silencing of MT1-MMP. As indicated in Fig. 6, the invasion ability of AGS cells was significantly inhibited following transfection with shRNA-2 $(\mathrm{P}<0.001)$. The results suggested that inhibition of MT1-MMP may suppress the invasion of cancer cells.

Suppression of genes associated with invasion. The mRNA expression levels of EMT-associated genes, including vimentin and epithelial cadherin (E-cadherin), were examined by RT-qPCR. Following transfection of the AGS cells with shRNA-2, the mRNA expression level of vimentin $(0.396 \pm 0.009)$ was significantly inhibited compared with the $\mathrm{NC}$ group $(0.661 \pm 0.040$; Fig. 7A; $\mathrm{P}<0.001)$. Conversely, the mRNA expression level of E-cadherin $(0.774 \pm 0.038)$ was significantly increased following the transfection of AGS cells with shRNA-2 compared with the NC group $(0.412 \pm 0.012$; Fig. 7B) $(\mathrm{P}<0.001)$. In addition, it was observed that the mRNA levels of vimentin and E-cadherin were significantly decreased in the NC (vimentin and E-cadherin, $\mathrm{P}<0.001$ ) and shRNA-2 group (vimentin, $\mathrm{P}<0.001$; E-cadherin, $\mathrm{P}<0.01$ ) compared with the blank group, which may due to a general cell response to transfection reagent toxicity. These results suggested that the suppression of MT1-MMP expression may decrease the invasion ability of gastric carcinoma cells.

\section{Discussion}

Gastric carcinoma, the second most common cause of tumor-associated mortality worldwide, contributes greatly to the global disease burden (20). Previously, with advances in surgical intervention and chemotherapy, the overall survival rate has been greatly improved. However, the 5 -year mortality rate for advanced gastric carcinoma remains as high as $30-50 \%$ (21). Therefore, extensive investigations are required for elucidating crucial molecules that participate in the pathogenesis of gastric carcinoma.

MT1-MMP, also known as MMP14, belongs to the MMPs family, which is correlated with invasion and metastasis of cancer cell (22). At present, MT1-MMP has been identified to be overexpressed in a variety of cancer tissues, including 
A Gastric carcinoma tissue

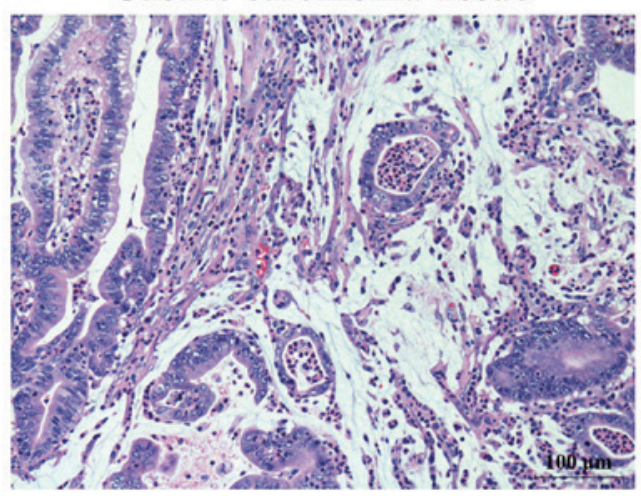

B Non-cancerous adjacent tissue

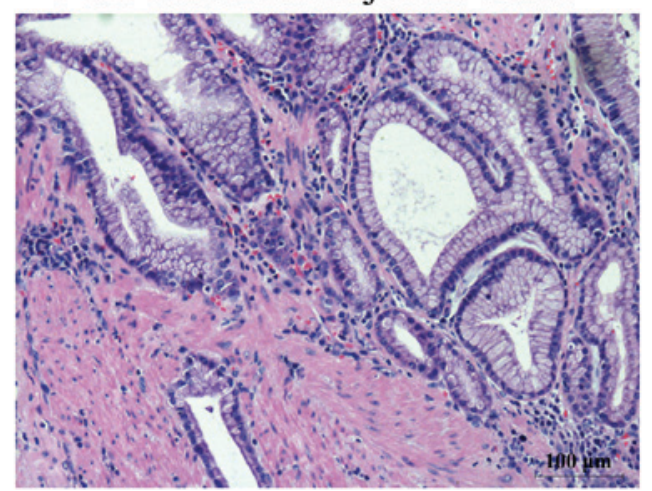

Figure 1. Hematoxylin-eosin staining. Staining of a (A) moderately differentiated gastric carcinoma tissue sample and (B) non-cancerous adjacent tissue sample. Magnification, x200. Scale bar, $100 \mu \mathrm{m}$.

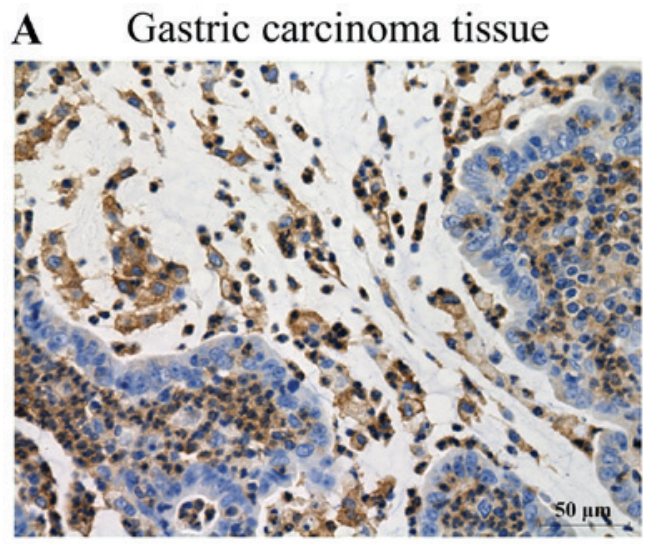

B Non-cancerous adjacent tissue

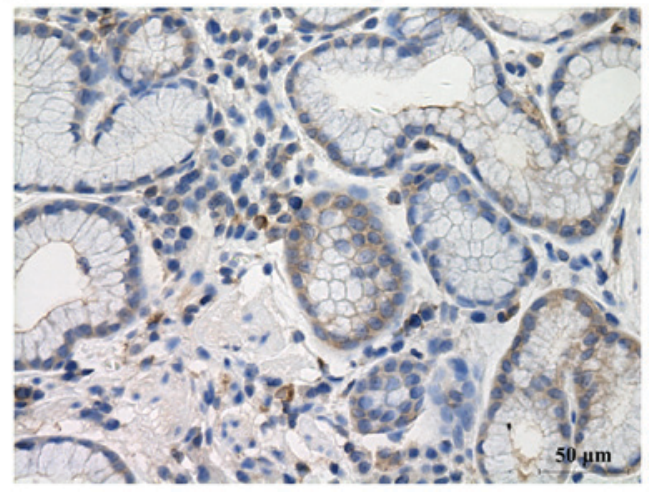

Figure 2. Expression of MT1-MMP in gastric carcinoma and non-cancerous adjacent tissues samples. (A) Intense staining was observed in gastric carcinoma tissues. This image is representative of a sample with moderate differentiation. Magnification, x400. (B) MT1-MMP protein expression was not detected in non-cancerous adjacent tissues. Magnification, $\mathrm{x} 400$. Scale bar, $50 \mu \mathrm{m}$. MT1-MMP, membrane type 1 matrix metalloproteinase.

colorectal and breast cancer $(23,24)$. A previous study demonstrated that MT1-MMP may promote breast tumor growth and angiogenesis through increasing the expression of vascular endothelial growth factor (VEGF) (25). Besides, the overexpression of MT1-MMP resulted in increased migration ability of esophageal squamous cell carcinomas (22). Corresponding results were also identified in the present study. In the present study, the tumor cell proliferation rate and invasion abilities were decreased by shRNA targeting MT1-MMP.

A previous study revealed that MT1-MMP was overexpressed in gastric carcinoma compared with that in adjacent tissues (26). The results from the present study also indicated that MT1-MMP was overexpressed in gastric carcinoma tissues through immunohistochemistry analysis. The overexpression of MT1-MMP was correlated with invasive lesions (22). Therefore, high expression levels of MT1-MMP may be associated with the invasion of gastric carcinoma. Furthermore, compared with the non-cancer-derived GES-1 cells, MT1-MMP was overexpressed in the cancer-derived cell line AGS cells. Subsequently, the present study screened an effective shRNA vector (shRNA-2) targeting MT1-MMP. Following transfection of the AGS cells with shRNA-2, the expression of MT1-MMP was markedly suppressed at mRNA and protein levels. Additionally, it was observed that inhibiting the expression of MT1-MMP was able to significantly decrease the proliferation rate and invasion ability of AGS cells. MT1-MMP is a critical protease that participates in the progress of cancer cell proliferation, migration and invasion (27). Tomari et al (28) revealed that the growth, invasion and metastasis of tumors was promoted by increasing MT1-MMP expression in tumor cells. Concomitantly, Pahwa et al (29) demonstrated that MT1-MMP was a crucial player in the growth and progression of melanoma. Therefore, these results indicated that MT1-MMP may promote gastric carcinoma cells growth and metastasis during the development of cancer.

In addition, the expression level of EMT-associated genes was examined, including vimentin and E-cadherin, to investigate the underlying mechanism of MT1-MMP in the progression of gastric carcinoma. Pang et al (22) suggested that MT1-MMP prompted esophageal squamous cell carcinoma invasion and metastasis by suppressing E-cadherin and subsequently inducing EMT. At present, a number of studies have demonstrated that EMT was associated with different types of tumors, including gastric, esophageal and hepatocellular carcinoma (22,30,31). Additionally, Sakamoto and Seiki (27) revealed that MT1-MMP was involved in the EMT progress of tumor development, by increasing the expression levels of hypoxia-inducible factors (32) and regulating the expression of 

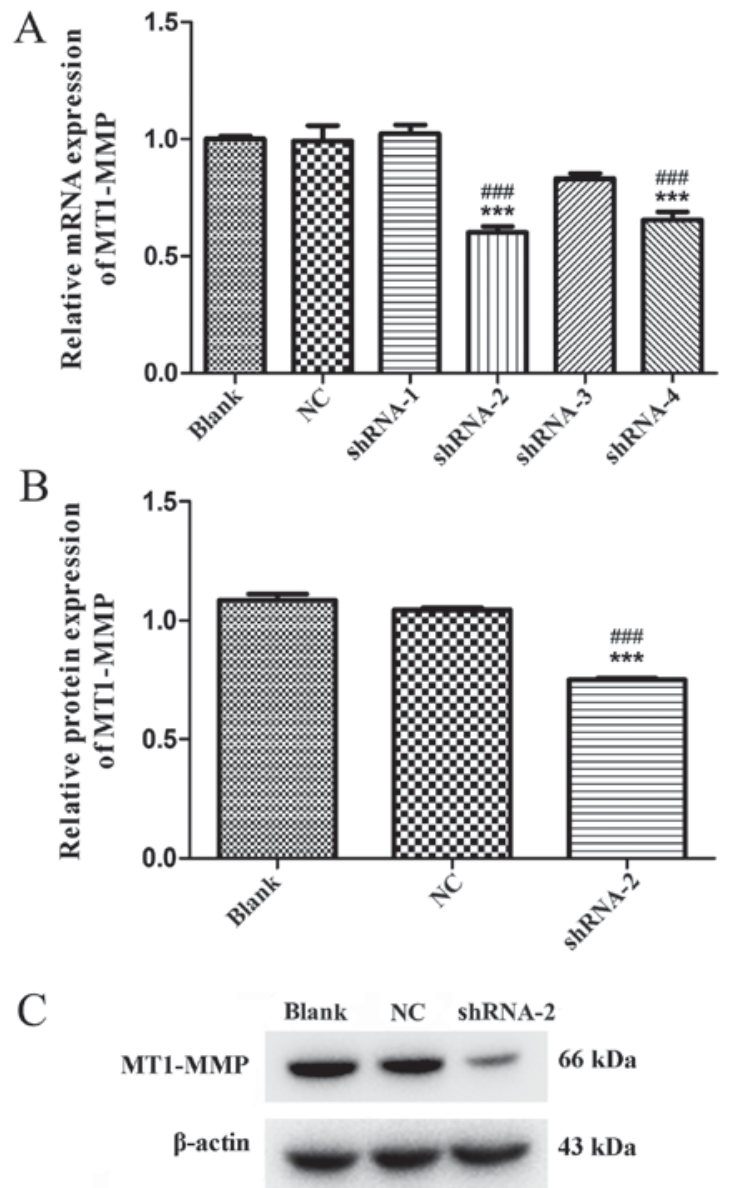

Figure 3. Verification and selection of recombinant shRNA. The interferential effects of 4 shRNAs were evaluated. (A) The effects of four shRNAs on the mRNA expression of MT1-MMP. (B) Quantitative data from western blot analysis. (C) Representative western blot analysis data of MT1-MMP expression in AGS cells transfected with shRNA-2. ${ }^{* * *} \mathrm{P}<0.001$ vs. blank group; ${ }^{\# \# \# P}<0.001$ vs. NC group $(\mathrm{n}=3)$. shRNA, short hairpin RNA; MT1-MMP, membrane type 1 matrix metalloproteinase; NC, negative control.

epithelial cell surface markers (22). The results of the present study suggested that the vimentin mRNA level was markedly decreased and the E-cadherin mRNA level was markedly increased following silencing of MT1-MMP. Concomitantly, differences in vimentin and E-cadherin expression between untreated AGS cells and empty pLKO.1-puro vector-treated AGS cells were observed in the present study, which may be due to a general cell response to transfection reagent toxicity, and require additional investigation. Taken together, we hypothesized that MT1-MMP was likely to mediate the invasion process via EMT, reflected by the altered expression of vimentin and E-cadherin. In summary, these results suggested that MT1-MMP may contribute to gastric carcinoma cell proliferation and invasion via regulating vimentin and E-cadherin expression. Future studies will investigate the molecular mechanisms underlying the effects of MT1-MMP1 on gastric cancer cell growth and invasion.

In conclusion, the present study confirmed that MT1-MMP was overexpressed in gastric carcinoma cells compared with non-cancerous adjacent tissues. Recombinant shRNA vectors targeting MT1-MMP successfully inhibited MT1-MMP expression in gastric carcinoma cells. In particular, silencing

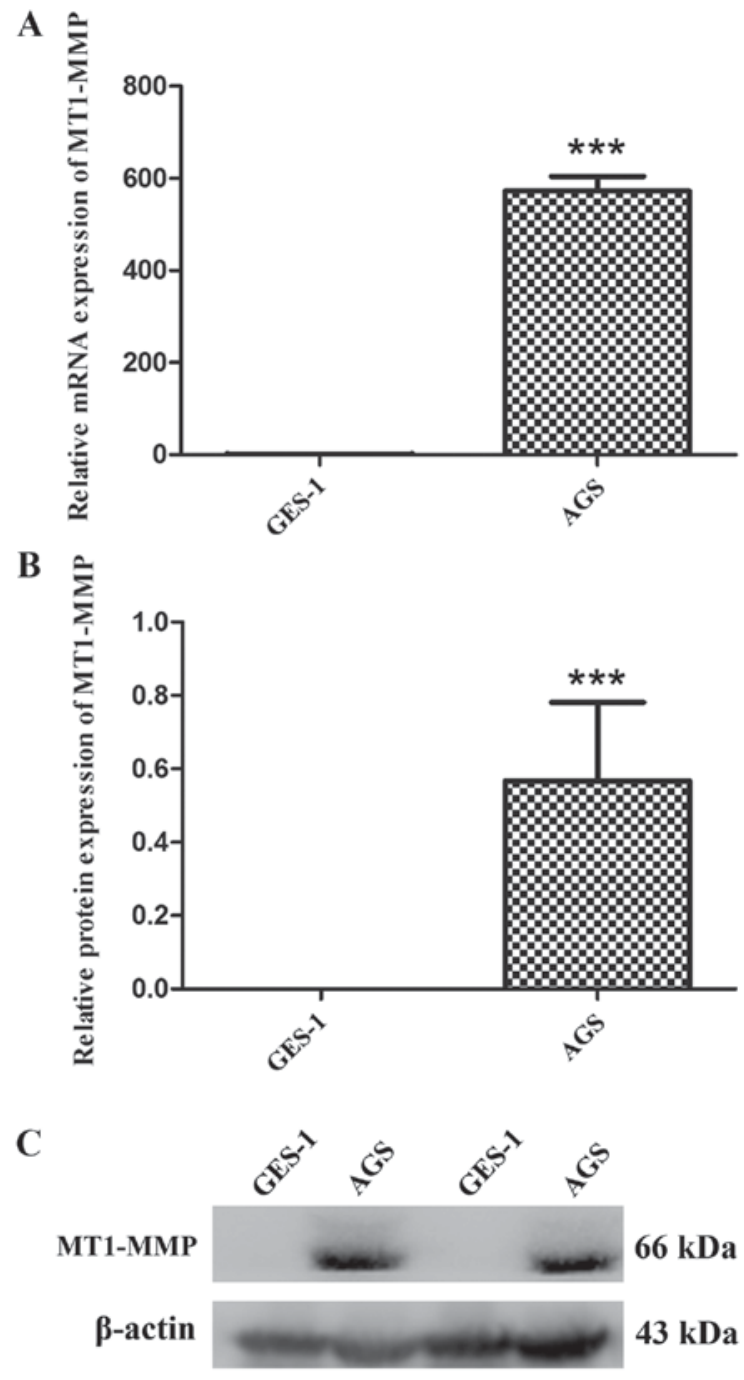

Figure 4. MT1-MMP expression levels in GES-1 and AGS cell lines. The expression level of MT1-MMP in GES-1 and AGS cells were evaluated. (A) Relative mRNA expression level of MT1-MMP in GES-1 and AGS cells. (B) Relative MT1-MMP protein expression level in GES-1 and AGS cells. (C) Representative western blot analysis of the protein expression of MT1-MMP in GES-1 and AGS cells. ${ }^{* * *} \mathrm{P}<0.001$ vs. GES-1 cells $(\mathrm{n}=3)$. MT1-MMP, membrane type 1 matrix metalloproteinase.

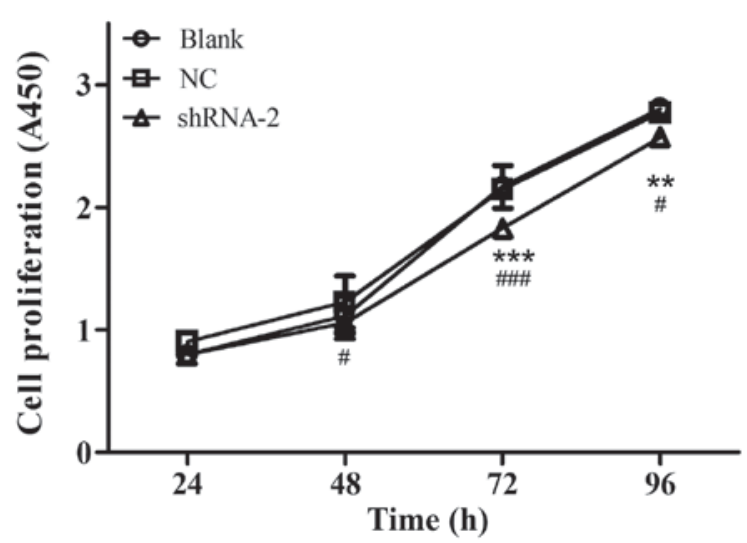

Figure 5. Cell Counting Kit-8 proliferation kit assay results of AGS cells transfected with shRNA-2. AGS were transfected with NC vectors (NC group) and shRNA-2. The proliferation rate of AGS was suppressed following transfection with shRNA-2. ${ }^{* *} \mathrm{P}<0.01$ and ${ }^{* * *} \mathrm{P}<0.001$ vs. blank group. ${ }^{\#} \mathrm{P}<0.05$ and ${ }^{\# \# "} \mathrm{P}<0.001$ vs. NC group ( $\left.\mathrm{n}=5\right)$. shRNA, short hairpin RNA; MT1-MMP, membrane type 1 matrix metalloproteinase; NC, negative control. 

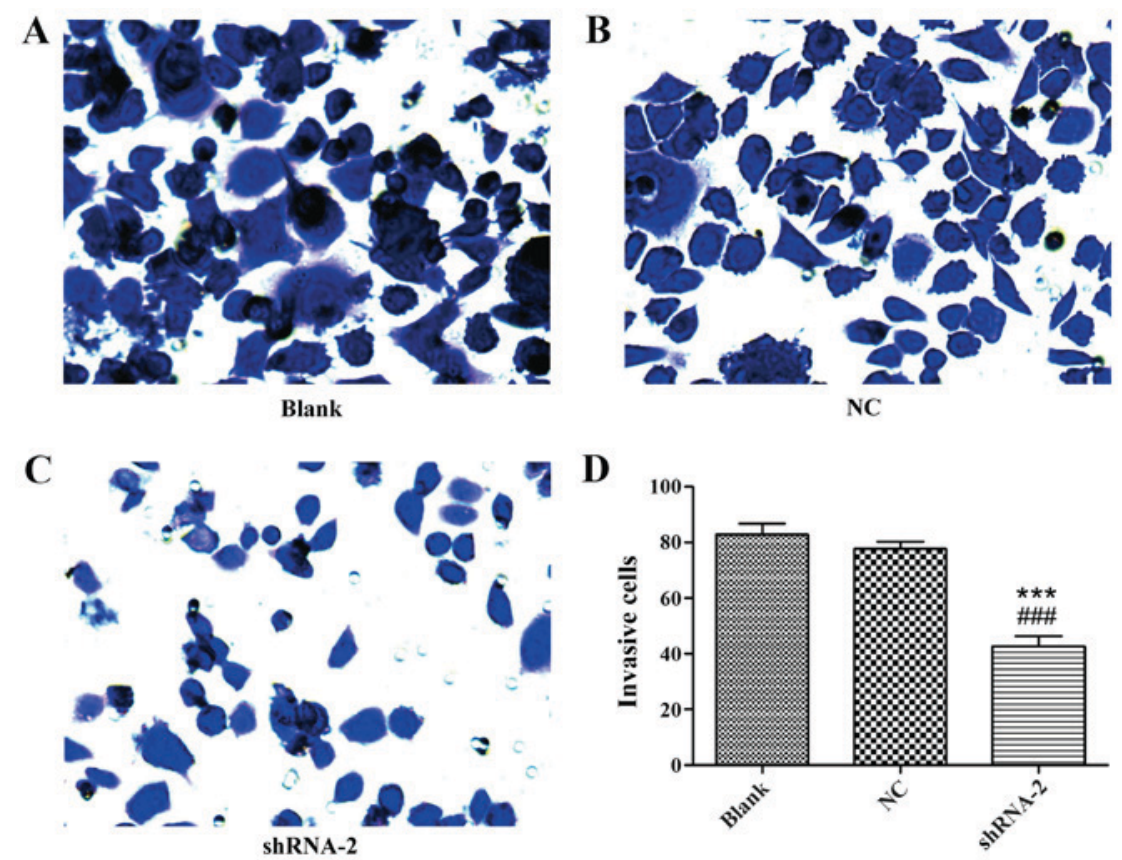

Figure 6. Transwell assays of AGS cells transfected with shRNA-2. The effect of MT1-MMP on the invasion ability of AGS cells was evaluated by Transwell assay. Transwell assay results of AGS cells transfected with (A) blank, (B) NC and (C) shRNA-2 vectors indicated that invasion was suppressed following shRNA-2 transfection. Magnification, $x 200$. (D) Quantitative data from the Transwell assay. ${ }^{* * *} \mathrm{P}<0.001$ vs. blank group; ${ }^{\# \# \#} \mathrm{P}<0.001 \mathrm{vs}$. NC group ( $\mathrm{n}=3$ ). shRNA, short hairpin RNA; MT1-MMP, membrane type 1 matrix metalloproteinase; NC, negative control.
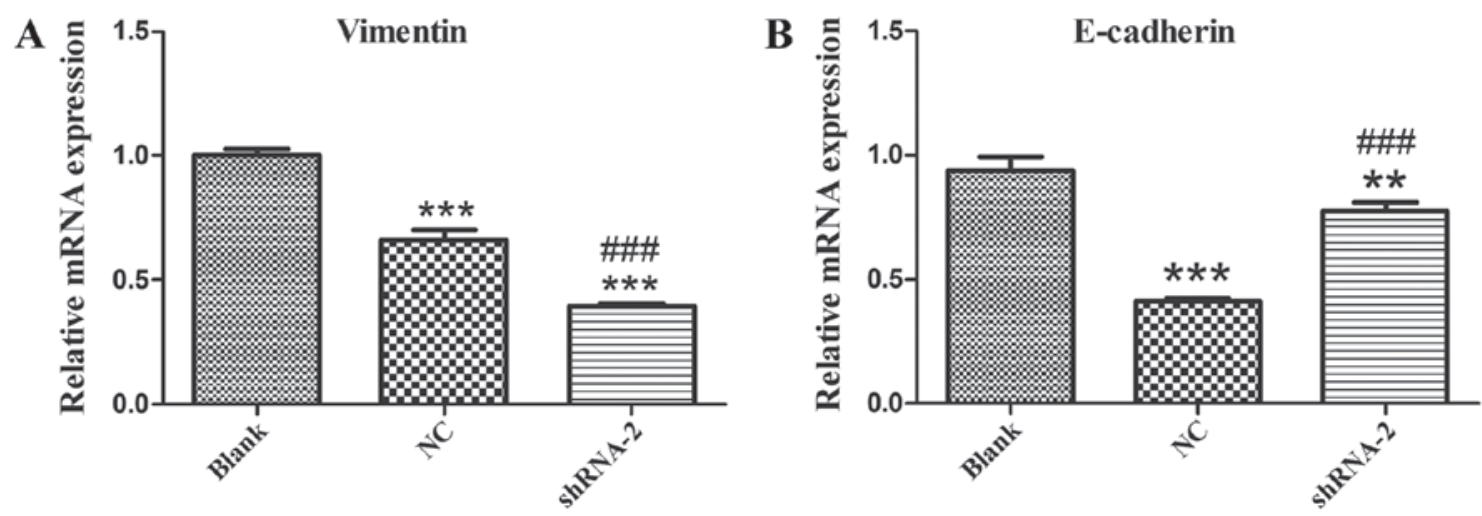

Figure 7. RT-qPCR results of vimentin and E-cadherin in AGS cells transfected with shRNA-2. Relative mRNA expression level of (A) vimentin and (B) E-cadherin of AGS cell transfected with shRNA-2 was measured by RT-qPCR. ${ }^{* *} \mathrm{P}<0.01$ and ${ }^{* * * *} \mathrm{P}<0.001$ vs. blank group; ${ }^{\# \# "} \mathrm{P}<0.001$ vs. NC group (n=3). shRNA, short hairpin RNA; RT-qPCR, reverse transcription quantitative polymerase chain reaction; E-cadherin, epithelial cadherin.

of MT1-MMP may inhibit cell proliferation and invasion via regulating the expression of EMT-associated genes, including vimentin and E-cadherin. In conclusion, the present study revealed that MT1-MMP may promote the proliferation and invasion of gastric carcinoma cells by regulating vimentin and E-cadherin expression.

\section{Acknowledgements}

Not applicable.

\section{Funding}

The present study was supported by Medicine and Health Science and Technology Plan Projects in Zhejiang Province (grant nos. 2014KYA013 and 2014KYA016).

\section{Availability of data and materials}

The datasets used and/or analyzed during the current study are available from the corresponding author on reasonable request.

\section{Authors' contributions}

JZ designed the study and performed the experiments. GL collected the data and performed the experiments. BL contributed to data analysis, data interpretation and discussion. All authors read and approved the final manuscript.

\section{Ethics approval and consent to participate}

The present study was approved by the Research Ethics Committee of the First Affiliated Hospital of Suzhou 
University and was performed according to ethical standards of the Declaration of Helsinki. All participants provided written consent for their clinical information to be used for scientific research.

\section{Patient consent for publication}

All participants provided written consent for their clinical information to be used for scientific research.

\section{Competing interests}

The authors declare that they have no competing interests.

\section{References}

1. Bray F, Ren JS, Masuyer E and Ferlay J: Global estimates of cancer prevalence for 27 sites in the adult population in 2008. Int J Cancer 132: 1133-1145, 2013.

2. Shah MA and Kelsen DP: Gastric cancer: A primer on the epidemiology and biology of the disease and an overview of the medical management of advanced disease. J Nati Compr Canc Netw 8: 437-447, 2010.

3. Siegel RL, Miller KD and Jemal A: Cancer statistics, 2015. CA Cancer J Clin 65: 5-29, 2015.

4. Camargo MC, Kim WH, Chiaravalli AM, Kim KM, Corvalan AH, Matsuo K, Yu J, Sung JJ, Herrera-Goepfert R, Meneses-Gonzalez F, et al: Improved survival of gastric cancer with tumour Epstein-Barr virus positivity: An international pooled analysis. Gut 63: 236-243, 2014.

5. Ueda Y, Fujishima H, Hirashita T, Shiroshita H, Etoh T, Inomata $\mathrm{M}$ and Shiraishi $\mathrm{N}$ : Clinical impact of small advanced gastric cancer $(\leq 40 \mathrm{~mm})$ in elderly patients: A retrospective cohort study. Int J Surg 45: 131-137, 2017.

6. Isobe Y, Nashimoto A, Akazawa K, Oda I, Hayashi K, Miyashiro I, Katai H, Tsujitani S, Kodera Y, Seto Y and Kaminishi M: Gastric cancer treatment in Japan: 2008 annual report of the JGCA nationwide registry. Gastric Cancer 14: 301-316, 2011.

7. Cheng TC, Din ZH, Su JH, Wu YJ and Liu CI: Sinulariolide suppresses cell migration and invasion by inhibiting matrix metalloproteinase-2/-9 and urokinase through the $\mathrm{PI} 3 \mathrm{~K} / \mathrm{AKT} / \mathrm{mTOR}$ signaling pathway in human bladder cancer cells. Marine drugs 15: E238, 2017.

8. D'Costa Z, Jones K, Azad A, van Stiphout R, Lim SY, Gomes AL, Kinchesh P, Smart SC, Gillies McKenna W, Buffa FM, et al: Gemcitabine-induced TIMP1 attenuates therapy response and promotes tumor growth and liver metastasis in pancreatic cancer. Cancer Res 77: 5952-5962, 2017.

9. Milovanovic J, Todorovic-Rakovic N and Abu Rabi Z: The role of interleukin 8 and matrix metalloproteinases 2 and 9 in breast cancer treated with tamoxifen. J BUON 22: 628-637, 2017.

10. Vos MC, Hollemans E, Ezendam N, Feijen H, Boll D, Pijlman B, van der Putten H, Klinkhamer P, van Kuppevelt TH, van der Wurff AA and Massuger LF: MMP-14 and CD44 in epithelial-to-mesenchymal transition (EMT) in ovarian cancer. J Ovarian Res 9: 53, 2016.

11. Robichaud N, Rincon SVD, Huor B, Alain T, Petruccelli LA, Hearnden J, Goncalves C, Grotegut S, Spruck CH, Furic L, et al: Phosphorylation of eIF4E promotes EMT and metastasis via translational control of SNAIL and MMP-3. Oncogene 34: 2032-2042, 2015.

12. Qin G, Luo M, Chen J, Dang Y, Chen G, Li L, Zeng J, Lu Y and Yang J: Reciprocal activation between MMP-8 and TGF- $\beta 1$ stimulates EMT and malignant progression of hepatocellular carcinoma. Cancer Lett 374: 85-95, 2016.

13. Zheng L, Li D, Xiang X, Tong L, Qi M, Pu J, Huang K and Tong Q: Methyl jasmonate abolishes the migration, invasion and angiogenesis of gastric cancer cells through down-regulation of matrix metalloproteinase 14. BMC Cancer 13: 74, 2013.

14. He L, Chu D, Li X, Zheng J, Liu S, Li J, Zhao Q and Ji G: Matrix metalloproteinase-14 is a negative prognostic marker for patients with gastric cancer. Dig Dis Sci 58: 1264-1270, 2013.
15. Ueda J, Kajita M, Suenaga N, Fujii K and Seiki M: Sequence-specific silencing of MT1-MMP expression suppresses tumor cell migration and invasion: Importance of MT1-MMP as a therapeutic target for invasive tumors. Oncogene 22: 8716-8722, 2003.

16. Berlth F, Bollschweiler E, Drebber U, Hoelscher AH and Moenig S: Pathohistological classification systems in gastric cancer: Diagnostic relevance and prognostic value. World J Gastroenterol 20: 5679-5684, 2014.

17. Pang L, Li Q, Wei C, Zou H, Li S, Cao W, He J, Zhou Y, Ju X, Lan J, et al: TGF- $\beta 1 /$ Smad signaling pathway regulates epithelial-to-mesenchymal transition in esophageal squamous cell carcinoma: in vitro and clinical analyses of cell lines and nomadic Kazakh patients from northwest Xinjiang, China. PLoS One 9: e112300, 2014.

18. Di Martino E, Wild CP, Rotimi O, Darnton JS, Olliver RJ and Hardie LJ: IGFBP-3 and IGFBP-10 (CYR61) up-regulation during the development of Barrett's oesophagus and associated oesophageal adenocarcinoma: potential biomarkers of disease risk. Biomarkers 11: 547-561, 2006.

19. Livak KJ and Schmittgen TD: Analysis of relative gene expression data using real-time quantitative PCR and the $2^{-\Delta \Delta C T}$ method. Methods 25: 402-408, 2001.

20. Torre LA, Bray F, Siegel RL, Ferlay J, Lortet-Tieulent J and Jemal A: Global cancer statistics, 2012. CA Cancer J Clini 65: 87-108, 2015.

21. Hamashima C, Shabana M, Okada K, Okamoto M and Osaki Y: Mortality reduction from gastric cancer by endoscopic and radiographic screening. Cancer Sci 106: 1744-1749, 2015.

22. Pang L, Li Q, Li S, He J, Cao W, Lan J, Sun B, Zou H, Wang C, Liu R, et al: Membrane type 1-matrix metalloproteinase induces epithelial-to-mesenchymal transition in esophageal squamous cell carcinoma: Observations from clinical and in vitro analyses. Sci Rep 6: 22179, 2016.

23. Jiang WG, Davies G, Martin TA, Parr C, Watkins G, Mason MD and Mansel RE: Expression of membrane type-1 matrix metalloproteinase, MT1-MMP in human breast cancer and its impact on invasiveness of breast cancer cells. Int J Mol Med 17: 583-590, 2006.

24. Shields MA, Dangi-Garimella S, Krantz SB, Bentrem DJ and Munshi HG: Pancreatic cancer cells respond to type I collagen by inducing snail expression to promote membrane type 1 matrix metalloproteinase-dependent collagen invasion. J Biol Chem 286: 10495-10504, 2011.

25. Sounni NE, Devy L, Hajitou A, Frankenne F, Munaut C, Gilles C, Deroanne C, Thompson EW, Foidart JM, and Noel A: MT1-MMP expression promotes tumor growth and angiogenesis through an up-regulation of vascular endothelial growth factor expression. FASEB J 16: 555-564, 2002.

26. Shen B, Zheng MQ, Xu XY, Mo FG, Zhang T and Feng JF: Expression of MT1-MMP and RECK protein in human gastric carcinoma. Zhonghua Shi Yan He Lin Chuang Bing Du Xue Za Zhi 25: 364-367, 2011 (In Chinese).

27. Sakamoto $\mathrm{T}$ and Seiki M: Integrated functions of membrane-type 1 matrix metalloproteinase in regulating cancer malignancy: Beyond a proteinase. Cancer Sci 108: 1095-1100, 2017.

28. Tomari T, Koshikawa N, Uematsu T, Shinkawa T, Hoshino D, Egawa N, Isobe T and Seiki M: High throughput analysis of proteins associating with a proinvasive MT1-MMP in human malignant melanoma A375 cells. Cancer Sci 100: 1284-1290, 2009.

29. Pahwa S, Stawikowski MJ and Fields GB: Monitoring and inhibiting MT1-MMP during cancer initiation and progression. Cancers 6: 416-435, 2014

30. Ouyang S, Zhu G, Ouyang L, Luo Y, Zhou R, Pan C, Bin J, Liao Y and Liao W: Bapx 1 mediates transforming growth factor- $\beta$ - induced epithelial-mesenchymal transition and promotes a malignancy phenotype of gastric cancer cells. Biochem Biophys Res Commun 486: 285-292, 2017.

31. Ji C, Liu H, Yin Q, Li H and Gao H: miR-93 enhances hepatocellular carcinoma invasion and metastasis by EMT via targeting PDCD4. Biotechnol Lett 1-9, 2017.

32. Sakamoto T and Seiki M: A membrane protease regulates energy production in macrophages by activating hypoxia-inducible factor-1 via a non-proteolytic mechanism. J Biol Chem 285: 29951-29964, 2010.

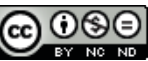

This work is licensed under a Creative Commons Attribution-NonCommercial-NoDerivatives 4.0 International (CC BY-NC-ND 4.0) License. 